\title{
Correlation between coronal hole and quiet Sun intensities: Evidence for continuous reconnection
}

\author{
K. P. Raju ${ }^{1, \star}$, B. J. I. Bromage ${ }^{1}$, S. A. Chapman ${ }^{1}$, and G. Del Zanna ${ }^{2}$ \\ ${ }^{1}$ Centre for Astrophysics, University of Central Lancashire, Preston, UK \\ e-mail: kpr@iiap.res.in \\ 2 DAMTP, University of Cambridge, Cambridge, UK
}

Received 14 October 2004 / Accepted 5 November 2004

\begin{abstract}
Intensities of 12 strong EUV emission lines in a coronal hole and the neighbouring quiet Sun region have been obtained from SOHO/CDS observations made in August and September 1996. The field of view is 1 arcmin wide by 4 arcmin, with a series of observations made at different spatial locations on the boundary of the north polar hole and its large equatorial extension, the "Elephant's Trunk". The formation temperatures of the observed lines vary from 0.083 MK to 1.10 MK and hence they represent increasing heights in the solar atmosphere from the upper chromosphere and transition region to the low corona. Line intensities in the vicinity of the coronal hole boundary were examined. For lines formed in the transition region, a correlation was found between intensities in the coronal hole and those in the adjacent quiet Sun region. However, for lines formed higher up in the low corona, the correlation gradually disappears. Moreover, it is found that in the lower transition region the coronal hole emission is brighter than that in the quiet Sun. We conclude that the correlation observed in the transition region implies that flux transport is not impeded by the presence of the coronal hole boundary, suggesting that reconnection is taking place which changes the status of the footpoints from closed to open and vice versa, maintaining the location of the boundary against the movement of the flux. The magnetic reconnection events provide energy in the corona which conducts down the field lines until it is absorbed in the transition region. The brightness of the coronal hole in the lower transition region lines can be explained if the energy conducted down the open field lines is able to penetrate to lower temperature levels than it does on the closed field of the quiet Sun region. This is possible because, for any given temperature in this range, the density in the coronal hole is lower than it is in the quiet Sun.
\end{abstract}

Key words. Sun: corona - Sun: transition region

\section{Introduction}

Coronal holes are cool, low density regions observed both at low latitudes and at the polar caps on the Sun. Their predominantly unipolar magnetic fields are open to the interplanetary region (Bohlin 1977), giving rise to high-speed solar wind that can lead to geomagnetic storms (Krieger et al. 1973). Because of their low density and temperature, they appear as dark regions in X-ray \& EUV images of the Sun. There are 3 broad categories of coronal holes: polar, non-polar and transient. Polar coronal holes have lifetimes of about 8 years, a significant fraction of the solar cycle itself. Non-polar coronal holes are usually associated with remnant active regions and may persist for many solar rotations. Transient coronal holes are associated with eruptive events like filament eruptions and Coronal Mass Ejections (CMEs) and have lifetimes around 1-2 days (Harvey $\&$ Recely 2002).

The evolution of coronal holes shows dependence on the solar cycle. Polar holes form about 1 to 3 years after the solar

* On leave from, Indian Institute of Astrophysics, Bangalore-560034, India. maximum. The area of the polar holes increases as solar activity decreases and reaches a maximum during solar minimum. Often they develop low latitude extensions which may cross the equator into the opposite hemisphere. As the cycle progresses, large scale movement of these open flux regions results from interaction between the open coronal hole field and the large, closed loops of the active region magnetic field. Magnetic reconnection between such open and closed field lines results in discrete changes in the connectivity of the field; flux at the footpoints of these field lines changes from closed to open and viceversa. Thus the coronal holes make discrete "jumps" from one location to another, gradually effecting transfer of one polarity of coronal hole from the north pole to the south pole via the active region belt, while the opposite polarity coronal hole field makes a similar journey from south to north. Thus as the cycle progresses polar holes shrink and more low-latitude holes appear. Polar holes disappear 1-2 years before solar maximum (Bromage et al. 2001; Harvey \& Recely 2002).

The boundaries of coronal holes are the interface between open field coronal hole regions and the surrounding closed field regions. Low latitude coronal holes may show quasi-rigid 
rotation and it has been suggested that magnetic reconnection must occur continuously at the boundary in order to maintain the coronal hole integrity (Kahler \& Hudson 2002). No evidence of such magnetic reconnection has been observed, however. It has also been suggested that magnetic reconnection at the boundaries may cause or facilitate some CMEs (Webb et al. 1978; Gonzalez et al. 1996). Although polar coronal holes are long-lived, their boundaries change continuously on time scales of hours. X-ray bright points and transient events like CMEs are thought to play a role (Kahler \& Moses 1990).

In recent years, it has been realized, and well characterized by observations made with the ULYSSES spacecraft, that two different kinds of solar wind exist: the fast steady solar wind originating from coronal holes and the slow unsteady solar wind arising from the streamer belt in low latitudes (Marsch 1999). Acceleration of the fast solar wind is not well-understood, though it is suggested that Alfven waves are a possible source of additional momentum. It is hence important to know the differences in physical properties of open and closed field regions and their variations throughout the solar atmosphere. Del Zanna \& Bromage (1999) obtained the electron density, temperature, abundances and the differential emission measure inside and outside the coronal hole. It has been generally found that the network is unaffected by the coronal hole phenomenon in the lower transition region. Gallagher et al. (1998) find that quiet Sun network is most prominent at temperatures around $\log T=5.6$. Stucki et al. (2002) have studied the differences between the quiet Sun and the coronal hole and find that the abrupt reduction of emission line intensities in the hole occurs at a temperature around $\log T=5.7$. Such studies also give insights into the evolution of network magnetic fields to the coronal field.

The present paper deals with spatial variations of the line intensities inside and outside a polar coronal hole and its equatorial extension, the Elephant's Trunk. A number of strong emission lines available in the CDS/NIS wavelength range have been selected to study the variations through the solar atmosphere from chromosphere to corona. Observations belong to the solar minimum period when coronal holes have their maximum coverage. In particular, we have examined the relationship between the intensities in a coronal hole and the adjacent quiet Sun region. The study is expected to give insights into the physical processes in the vicinity of a coronal hole boundary.

\section{Data}

The observations were part of a programme to study the evolution of coronal holes throughout the solar cycle. The present study uses SOHO/CDS observations made during the Whole Sun Month (August-September 1996). Details of the observations may be seen in Del Zanna (1999), Bromage et al. (2000). Though there are a few tens of emission lines available in 16 wavelength windows of the CDS study, we have selected only 12 of the strongest lines for the present analysis; these are listed in Table 1 . The line parameters as well as the line formation temperature may be seen in the table; the latter is obtained from the CHIANTI (Dere et al. 1997) atomic database
Table 1. Details of emission lines used and the results of the analysis. Columns 2-4 give the emission line parameters. Columns 5 and 6 give the correlation coefficient $q$ and the probability $P$ that the correlation coefficient arises from a random distribution respectively. Column 7 gives the slope of the straight line fit and its error.

\begin{tabular}{lllllll}
\hline \hline No. & Ion & $\begin{array}{l}\lambda \\
{[\AA]}\end{array}$ & $\begin{array}{l}T \\
{[\mathrm{MK}]}\end{array}$ & $q$ & $P$ & slope \\
\hline 1 & HeII(2) & 607.56 & 0.083 & 0.56 & 0.01 & $0.60 \pm 0.02$ \\
2 & OIII & 599.59 & 0.11 & 0.86 & 0.00 & $0.59 \pm 0.01$ \\
3 & OIV & 608.40 & 0.19 & 0.83 & 0.00 & $0.53 \pm 0.01$ \\
4 & OV & 629.73 & 0.25 & 0.84 & 0.00 & $0.68 \pm 0.01$ \\
5 & NeVI & 562.80 & 0.43 & 0.83 & 0.00 & $0.78 \pm 0.01$ \\
6 & NeVII & 561.73 & 0.52 & 0.74 & 0.00 & $0.93 \pm 0.01$ \\
7 & CaX & 557.76 & 0.66 & 0.06 & 0.77 & \\
8 & MgVIII & 315.01 & 0.81 & 0.29 & 0.18 & $0.62 \pm 0.02$ \\
9 & SiVIII & 316.22 & 0.81 & 0.40 & 0.06 & $0.63 \pm 0.02$ \\
10 & MgIX & 368.06 & 0.95 & 0.07 & 0.76 & \\
11 & SiIX & 349.9 & 1.07 & -0.13 & 0.55 & \\
12 & MgX & 624.94 & 1.10 & 0.06 & 0.80 & \\
\hline
\end{tabular}

version 4.02. The line formation temperature is taken as the peak of the contribution functions plotted in Fig. 1.

The EIT $195 \AA$ image and CDS raster area are shown in Fig. 2. The slit used was $240^{\prime \prime} \times 4^{\prime \prime}$ and each raster covered an area $240^{\prime \prime} \times 20^{\prime \prime} .3$ rasters were then combined to form a composite $240^{\prime \prime} \times 60^{\prime \prime}$ window on the Sun. The window covers part of the coronal hole, the hole boundary and the adjacent quiet region. Rasters from NIS 1 were repeated three times and averaged because of its lower sensitivity. Even for the strong emission lines selected, their low intensity in coronal holes is a problem, and hence both spatial and temporal averaging have been used to improve the signal-to-noise ratio. The various averaging steps have reduced the spatial resolution to about $20^{\prime \prime}$ and the temporal resolution to about an hour.

The intensity profiles were obtained using the standard CDS calibration available in Solar SoftWare (SSW). They were then fitted with single/multiple Gaussians to obtain the individual components. Only those line profiles with a signal-to-noise ratio above 10 were considered. The integrated intensities of the lines were obtained from the relationship

$I=\frac{1}{2} \sqrt{\frac{\pi}{\ln 2}} A w_{\mathrm{f}}\left[\mathrm{W} \mathrm{m}^{-2} \mathrm{sr}^{-1}\right]$

where $A$ is the amplitude of the emission line and $w_{\mathrm{f}}$ is the full width at half maximum ( $F W H M)$.

\section{Results}

The observations cover different spatial locations on the north polar hole and its equatorial extension, known as the Elephant's Trunk. Altogether 24 observations were analysed, out of which 17 were in the polar regions near the central meridian, and seven were from the equatorial extension. The coronal hole and the quiet Sun region were identified in the windows based on the intensity of the $\operatorname{MgX} 625 \AA$ line. Two intensity contour levels which represent the coronal hole and the quiet Sun region were found by a trial and error method and by comparing 


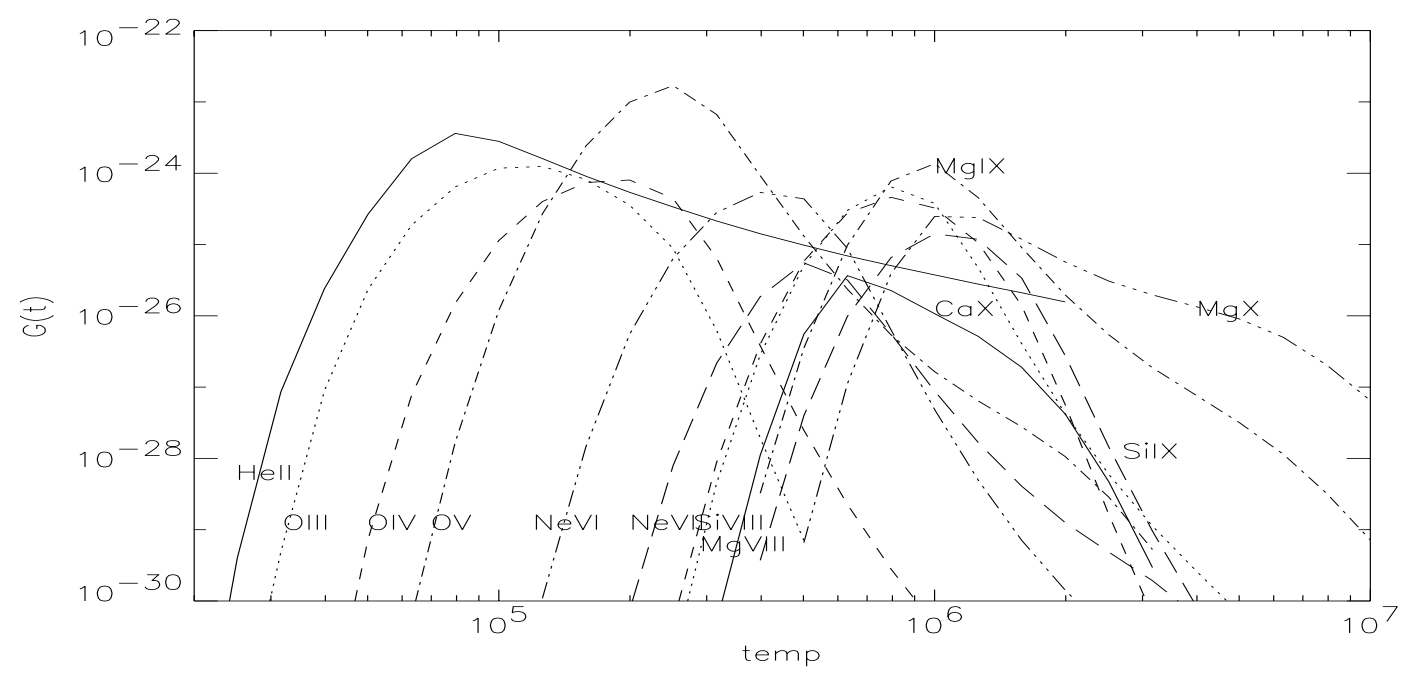

Fig. 1. Contribution functions plotted as a function of temperature (from CHIANTI version 4.02).

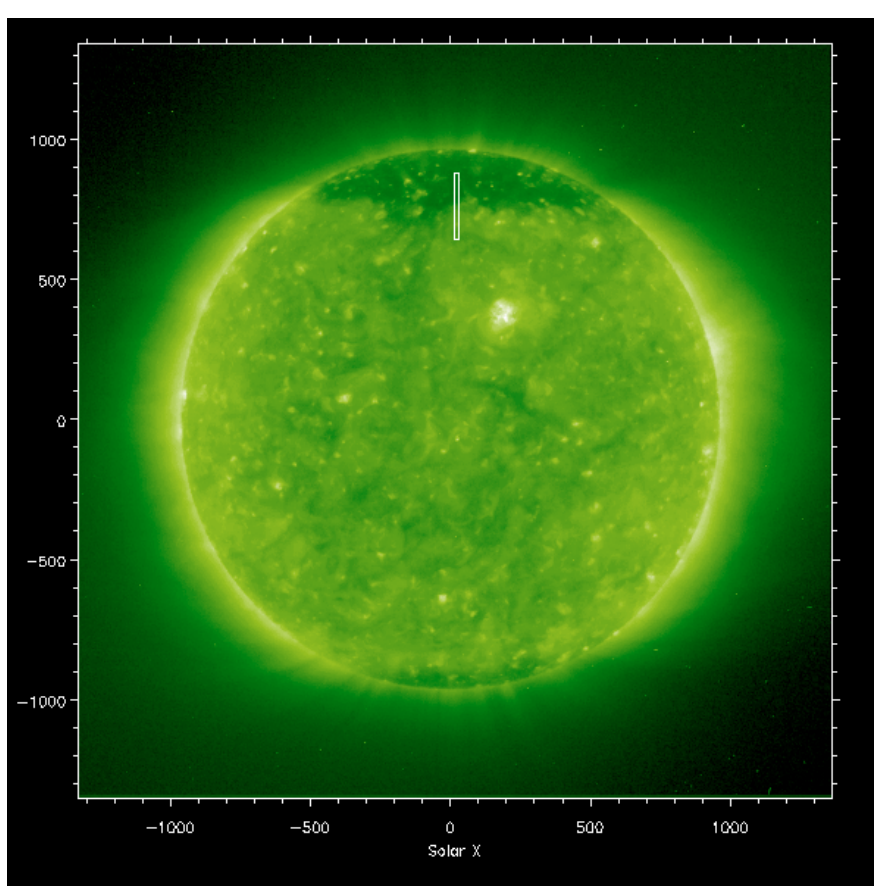

Fig. 2. EIT $195 \AA$ image and the raster position.

them with the EIT images. Figure 3 shows images formed from each of the 12 strongest emission lines, from one such observation. The two contour levels which represent the coronal hole and the quiet region are also plotted in the image. The area between contour 1 and 2 is a buffer zone which represents the hole boundary. It can be seen that the network is dominant in lower transition region lines but gradually disappears in the corona.

After identifying the coronal hole and the quiet Sun region for each window, we have examined the intensity variations in the two regions. There appeared to be a correlation between the intensity in the coronal hole and that of the adjacent quiet Sun region, for some of the wavelengths examined. A correlation analysis was performed; the results are summarised in Table 1 and Fig. 4. In each case, the mean value of the intensity of the coronal hole region and the adjacent quiet Sun region, on the other side of the boundary were obtained. Scatter plots were produced from these means, plotting quiet Sun intensity against coronal hole intensity for each data set. These are displayed in Fig. 4, with linear least-squares fits superimposed in those cases where the correlation was found to be significant. The slope of the fitted line is given in Table 1. The correlation coefficient and the probability that the correlation arises from a random distribution are also given in Table 1. It may be seen that there is a good linear correlation between coronal hole and quiet Sun line intensities for the transition region lines. The correlation gradually decreases upwards until, at a temperature around $1 \mathrm{MK}$, the correlation completely disappears.

In Fig. 4, we have also plotted a line of equal intensity (dots) in those cases where the correlation is significant. It can be seen that coronal hole is typically brighter than the adjacent quiet region in the lower transition region lines OIII $600 \AA$ and OIV $608 \AA$. For OV $630 \AA$, the two regions appear to be of equal brightness, whilst in hotter lines the quiet Sun region intensities are brighter than those in the coronal hole. Stucki et al. (2000) find some evidence for enhanced network in coronal holes, though their later paper with more samples (Stucki et al. 2002) gives a negative result. However, one possible explanation is suggested by the evidence in Fig. 4 of some intensity dependence in the relationship between quiet Sun and coronal hole brightness seen in the OIII and OIV lines (see the Discussion section, below). Here, the coronal hole data sets with the lowest intensity do not appear to be significantly brighter than the adjacent quiet Sun region. Thus, the relationship inferred from these data will depend on the brightness of the particular observation, and so could vary from one data set to another. Only with a large number of examples, such as have been studied here, can the distribution of the effect, as a function of intensity, be seen.

\section{Discussion}

To summarise, the main results obtained are: (1) there exists a correlation between the intensity found in a coronal hole and that in the quiet Sun region on the other side of the boundary 

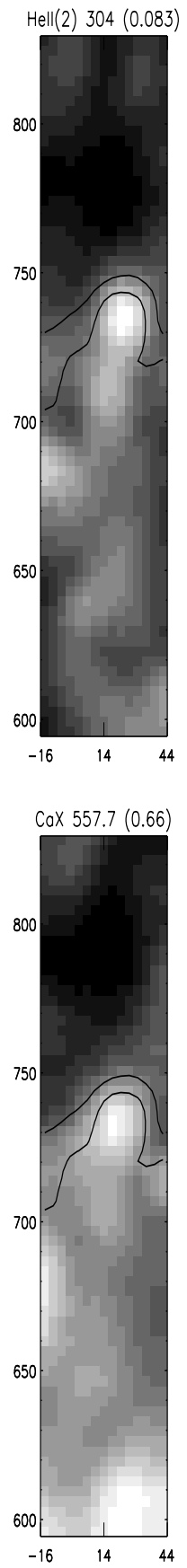
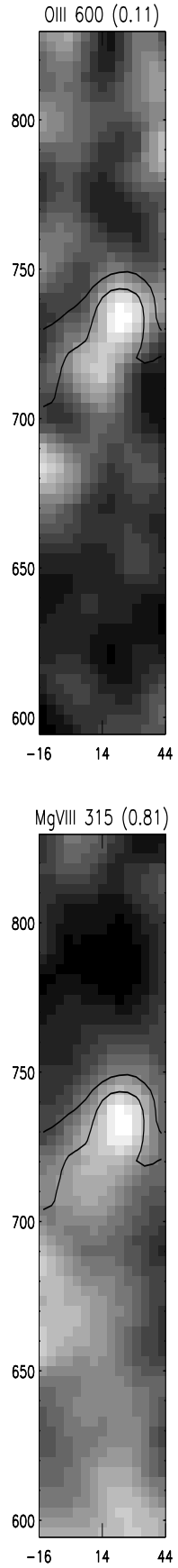
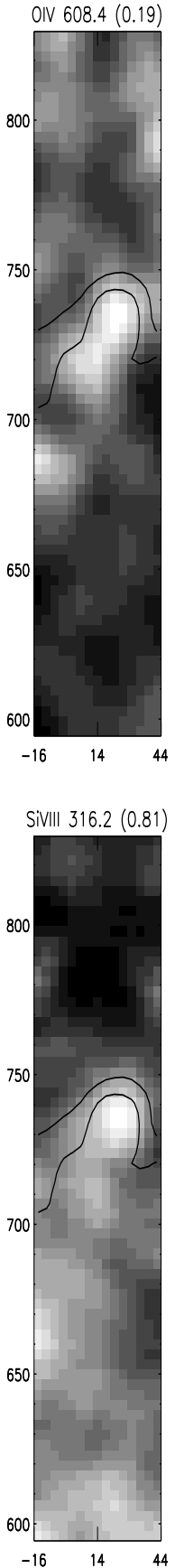
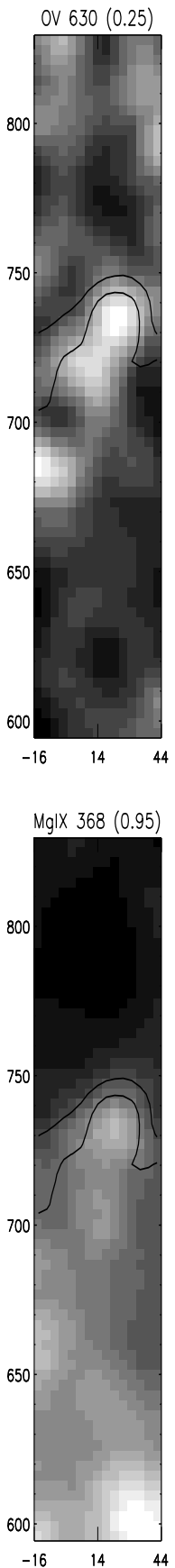
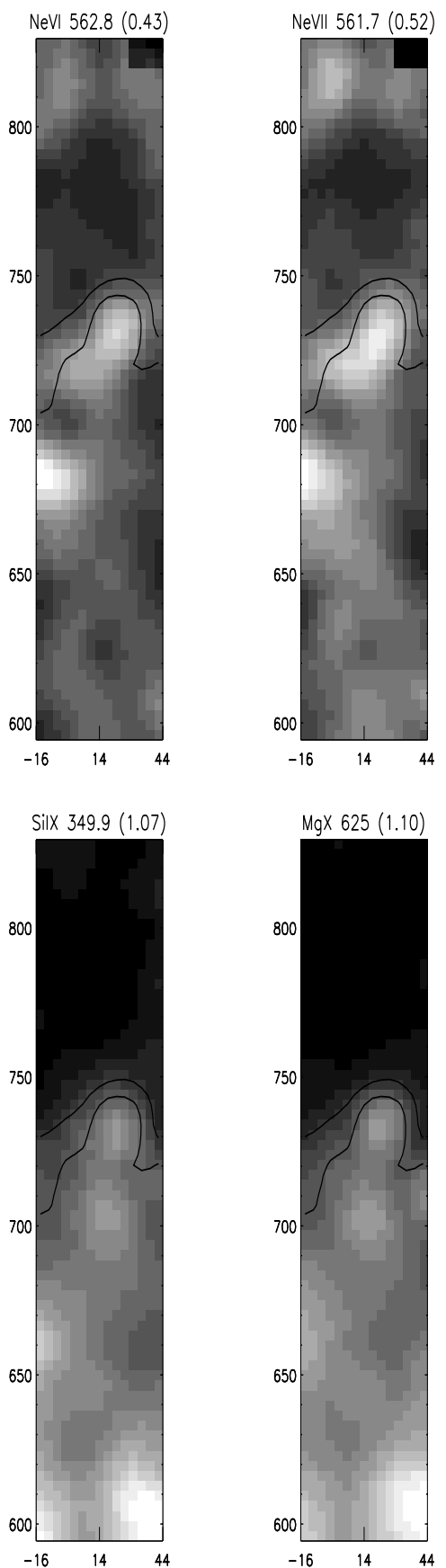

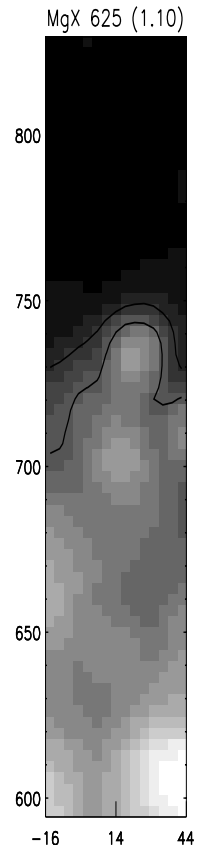

Fig. 3. Observed window in 12 emission lines. Line formation temperatures $[\mathrm{MK}]$ are given in the bracket. Contours represent coronal hole (upper part) and quiet Sun (lower part).

between the two. This exists for lines in which the chromospheric network can be seen (chromospheric and transition region lines); (2) the network is brighter in the coronal hole than in the corresponding quiet Sun region when seen in the lower transition region.

The magnetic flux on the surface of the Sun is mainly transported by the supergranular convection, differential rotation, and meridional circulation. In this way, flux associated with an emerging active region is gradually dispersed around the network boundaries and also in latitude and longitude as the active region evolves and fades. It has been clear since Skylab observations first revealed large low-latitude coronal holes that many of these appear to rotate quasi-rigidly, at a faster rate than the underlying photosphere at mid-latitudes. This was later confirmed in a study by Insley et al. (1995). It implies that the boundary of such extended coronal holes must be moving relative to the underlying photospheric flux. For a correlation to exist between regions on either side of a coronal hole boundary, this flux transport would have to take place regardless of whether the flux is open or closed. However, this suggests that as flux moves across the coronal hole boundary it must change from closed to open, otherwise the boundary would have to move along with the flux. This phenomenon occurs both for polar holes where the meridional circulation disperses the flux 

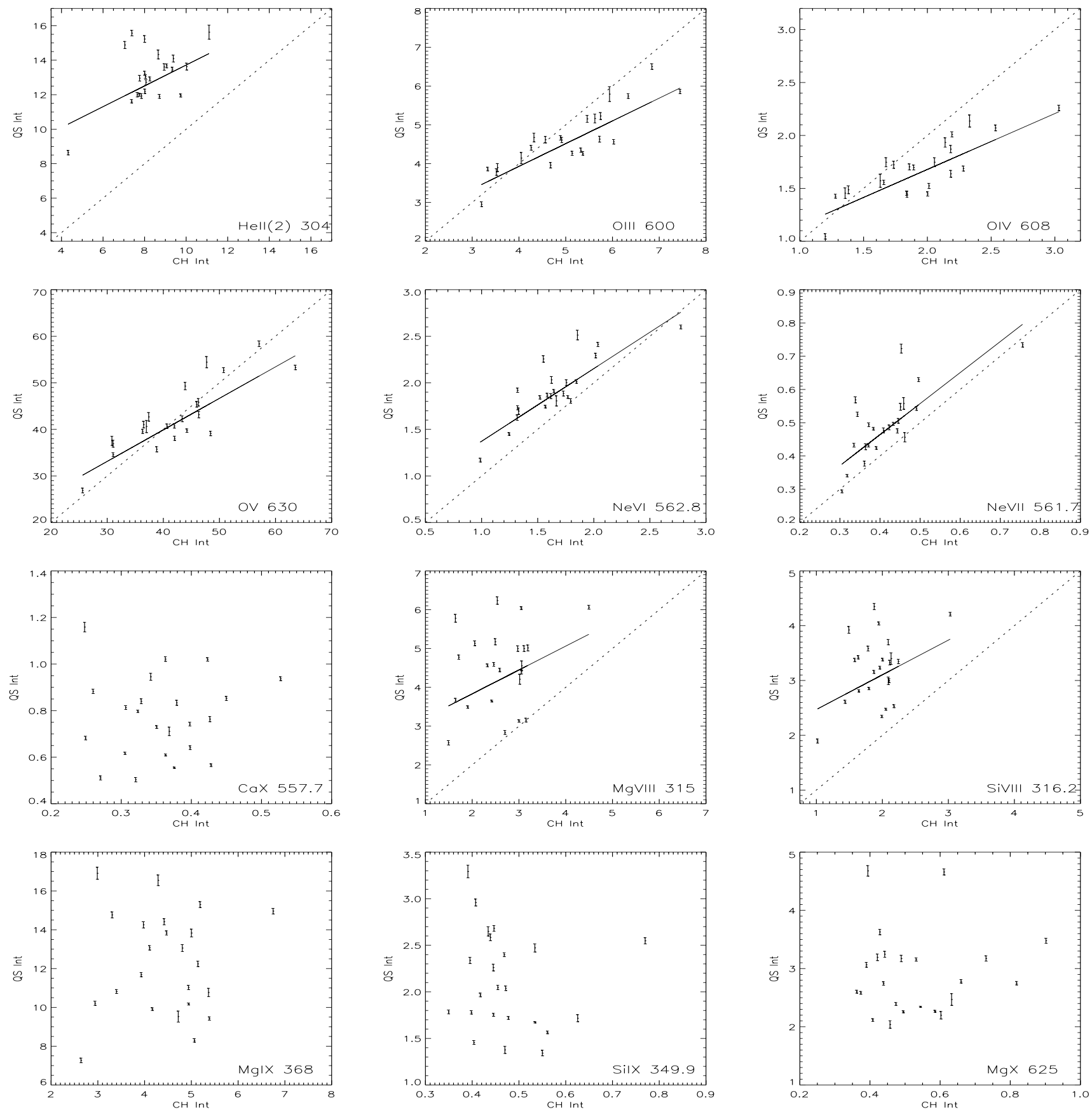

Fig. 4. Quiet Sun intensities are plotted against coronal hole intensities. Intensity units are $\left[10^{-3} \mathrm{~W} \mathrm{~m}^{-2} \mathrm{sr}^{-1}\right]$. Solid line represents a straight line fit. Fit parameters are given in Table 1. Dotted line represents the line of equal intensity.

in latitude and for N-S oriented coronal holes exhibiting quasirigid rotation. For the latter the question of how such holes can rotate rigidly while maintaining continuity with differentially rotating photospheric plasma has been a puzzle for some time. It has been suggested (Wang \& Sheeley 1993) that continuous reconnection occurs across the boundary in response to this conflict. This could also explain how the flux transport can take place across a coronal hole boundary, maintaining a relation between the flux in the two regions and hence a correlation in intensity between the two. Such reconnection occurring between the adjacent open and closed field lines in the coronal hole boundary region will have the effect of changing the footpoints of the open field. Thus, as the flux moves from quiet Sun across the boundary to the coronal hole, the associated field lines reconnect and change from closed to open.

The reconnection occurs above, in the corona where plasma beta is low and conductivity is high. Energy from each event will then conduct down the field lines to the transition region. We may be able to explain our second result here, since the field lines within the coronal hole have lower density plasma associated with them than in the quiet Sun (Del Zanna \& Bromage 1999). Here, the energy can travel further before being halted in the lower transition region, dumping most of the energy here, producing enhanced spectral emission 
(brightenings), whereas conduction along the closed field lines will be deposited in the regions of higher temperature, in the transition region and lower corona. As a result, at lower transition region levels, more emission is produced in the coronal hole than in the nearby quiet Sun region. More specifically, for the OIII and OIV lines, it is clear that those observations at higher intensities are brighter in the coronal hole than in the quiet Sun. However, it can be seen from Fig. 4 that there appears to be some dependence on intensity, such that the lowest intensity samples tend to lie close to the dotted line, suggesting that for such observations the two regions are of equal brightness. This is consistent with the argument that the enhanced emission in the coronal hole region is a result of downward conduction of energy from reconnection events in the corona: the less intense data sets are regions for which few reconnection events are currently occurring and so the brightenings due to conduction from the corona are fewer or non-existent. Without these brightenings, the mechanism for excess intensity in the coronal hole is not present. It is interesting to note, moreover, that under such circumstances, the two adjacent regions, either side of the coronal hole boundary, are equally bright, suggesting that at these temperatures $(0.1-0.2 \mathrm{MK})$ the "quiescent emission" is the same from open and closed magnetic field regions.

The situation is further complicated by the fact that the transition region is not at the same altitude in the coronal hole as it is in the quiet Sun areas (Gabriel 1976). However, our observations are comparing intensities from the same lines in the two regions, so the temperature of formation is the same even though the emitting plasma may not be at the same height in the solar atmosphere. For plasma at the same temperature, the density is lower in the coronal hole than in the quiet Sun.

The loss of correlation at the coronal level is related to the disappearance of the network which dominates the emission at lower levels. The coronal emission here is from more uniformly distributed plasma associated with the low-beta magnetic field and the intensity now becomes related to whether the field is open or closed, with more plasma and higher emission in the closed-field regions.

There are two clear exceptions to the general behaviour: the He II $304 \AA$ line and the CaX $558 \AA$ line. The anomalous behaviour of the He line is well known. Owing to the resonance scattering mechanism of line emission and large optical depth, it shows the characteristics of both chromosphere and the corona (Gallagher et al. 1998; Jordan et al. 2001). The calcium line behaves more like a coronal line which is not typical of its line formation temperature. This is probably due to the asymmetrical high-temperature wing of its contribution function (see Fig. 1). This conjecture is also supported by the behaviour of MgVIII $315 \AA$ and SiVIII $316 \AA$ lines. Both have higher formation temperatures but continue to show the gradual behaviour. Stucki et al. (2002) find that the transition from lines not influenced by the coronal hole and those significantly weakened by it is surprisingly narrow in temperature. They find that NeVII line is unaffected by the hole while $\mathrm{Ca} \mathrm{X}$ is weakened to less than half its quiet Sun strength. This is probably better explained if we accept the anomalous behaviour of the $\mathrm{Ca} \mathrm{X}$ line. Hence our findings are in variance with the above paper, and further suggest that the behaviour of the emission line is not merely decided by the peak of its contribution function but also by its detailed shape.

\section{Conclusions}

The intensities of 12 strong EUV emission lines in a coronal hole and the quiet region just outside the hole have been obtained during the solar minimum period. The formation temperatures of the observed lines vary from from $0.083 \mathrm{MK}$ to $1.10 \mathrm{MK}$ and hence they represent increasing heights in the solar atmosphere from the transition region to the corona. It has been found that the intensities of the coronal hole and the quiet region outside it are correlated for the transition region lines. The correlation disappears at the coronal level. The positive correlation is explained on the basis of magnetic flux transport by the supergranulation, differential rotation, and meridional circulation. However, a requirement for correlation across the coronal hole boundary is that continuous reconnection is taking place here. Thus the correlation that we have found supports the hypothesis of continuous reconnection at coronal hole boundaries. There are two clear exceptions to the general behaviour: the He II $304 \AA$ line and the Ca X $558 \AA$ Aline. The anomalous behaviour of the He line is expected. The calcium line behaves more like a coronal line which is not typical of its line formation temperature. This is probably due to the asymmetrical high-temperature wing of its contribution function. It has also been found that the coronal hole is brighter than the adjacent quiet Sun region in the lower transition region lines OIII $600 \AA$ and OIV $608 \AA$. The enhanced brightness in the coronal holes can be explained as resulting from energy conducting down the field lines from coronal levels. This will be able to penetrate to lower temperature regions here than in the quiet Sun, because of the lower density in the coronal hole at any given temperature. The effect becomes more pronounced as the particles descend to levels where the temperature is below the peak network energy deposition level determined by Gallagher, of $0.52 \mathrm{MK}$ (the temperature of peak emission from Ne VII).

The distribution of intensity in the transition region lines is determined by the magnetic flux, which in turn is driven to the edges of the chromospheric network cells by the supergranular convection. It is further dispersed in latitude and longitude by the meridional flow and differential rotation. Thus the flux originating from active regions becomes spread out and diffused over time, but predominantly located in the network boundaries. We conclude that correlation between the quiet Sun and coronal hole emission seen in the transition region implies that the flux transport is not impeded by the boundary between the two regions, and therefore reconnection must be taking place here. In the corona, the emission is no longer dominated by the network structure, but by small loops which exist mainly in the quiet Sun region, with very few in the largely monopolar field of the coronal holes. This leads to the breakdown in the correlation in the low corona and above.

Acknowledgements. K. P. Raju is supported by a PPARC research grant. Data provided courtesy of SOHO/CDS consortium. SOHO is 
a project of international cooperation between ESA and NASA. The authors are grateful to the CDS project team and the EIT consortium for their support.

\section{References}

Bohlin, J. D. 1977, in Coronal Holes and High Speed Wind Streams, ed. J. B. Zirker (Boulder, Col.: Colorado Associated Press)

Bromage, B. J. J., Alexander, D., Breen, A., et al. 2000, Sol. Phys., 193, 181

Bromage, B. J. I., Browning, P. K., \& Clegg, J. R. 2001, Space Sci. Rev., 97, 13

Del Zanna, G. 1999, Ph.D. Thesis, Univ. of Central Lancashire

Del Zanna, G., \& Bromage, B. J. I. 1999, JGR, 104, 9753

Del Zanna, G., \& Bromage, B. J. I. 1999, Space Sci. Rev., 87, 169

Dere, K. P., Landi, E., Mason, H. E., Monsignori Fossi, B. C., \& Young, P. R. 1997, A\&AS, 125, 149

Gabriel, A. H. 1976, Phil. Trans. R. Soc. Lond. A, 281, 339
Gallagher, P. T., Phillips, K. J. H., Harra-Murnion, L. K., \& Keenan, F. P. 1998, A\&A, 335, 733

Gonzalez, W. D., Tsurutani, B. T., McIntosh, P. S., \& Clúa de Gonzalez, A. L. 1996, GeoRL, 23, 2577

Harvey, K. L., \& Recely, F. 2002, Sol. Phys., 211, 31

Insley, J. E., Moore, V., \& Harrison, R. A. 1995, Sol. Phys., 160,1

Jordan, C., Macpherson, K. P., \& Smith, G. R. 2001, MNRAS, 328, 1098J

Kahler, S. W., \& Hudson, H. S. 2002, ApJ, 574, 467

Kahler, S. W., \& Moses, D. 1990, ApJ, 362, 728

Krieger, A. S., Timothy, A. F., \& Roelof, E. C. 1973, Sol. Phys., 29, 505

Marsch, E. 1999, Space Sci. Rev., 87, 1

Stucki, K., Solanki, S. K., Schühle, U., et al. 2000, A\&A, 363, 1145

Stucki, K., Solanki, S. K., Pike, C. D., et al. 2002, A\&A, 381, 653

Wang, Y.-M., \& Sheeley, N. R., Jr., D. 1993, ApJ, 414, 916

Webb, D. F., Nolte, J. T., Solodyna, C. V., \& McIntosh, P. S. 1978, Sol. Phys., 58, 389 